and factor groups. The final Chapter 9 deals with class formations and the associated Weil groups. This is the abstract scheme that embodies the main cohomological features of class field theory.

As is natural for this type of report, it assumes that the reader is familiar with the basic notions and techniques of general homological algebra, and it leaves a fair amount of detail for the reader to fill in. For the specialist, this book is an extremely valuable complement to the existing literature on the subject.

\title{
G. HOCHSCHILD
}

Cohomology operations and applications in homotopy theory by Robert E. Mosher and Martin C. Tangora. Harper's Series in Modern Mathematics, Harper and Row, New York, 1968. $x+214$ pp. $\$ 12.95$.

This book is based on a revised version of notes taken by the second author during a course given by the first. According to the author's preface the book is intended for advanced graduate students having considerable knowledge about cohomology and some familiarity with homotopy groups. The book concentrates on the mod 2 theory and only gives references for the mod $p$ results.

The authors begin with a definition of the Steenrod squares which is one of the several different definitions originally due to Steenrod. All the fundamental properties of the squares except uniqueness are proved. This is followed immediately by an application to vector fields on spheres and the Hopf invariant. Spectral sequences are introduced in order to state Serre's theorem. No proof is given. This material is applied to calculate $H^{*}(K(\pi, n))$ where $\pi=Z$ or $Z_{2}$. Serre's theory of classes of Abelian groups is introduced and used to facilitate the calculation of some homotopy groups of $S^{n}$. These calculations are used to illustrate some of the theory. The book finishes with short treatments of Postnikov towers, higher cohomology operations and the Adams spectral sequence.

The book is well written and interesting to read. The main criticism of the book is in its emphasis. It seems to me that any treatment of this sort should give more play to the Adams spectral sequence both for the calculations of homotopy groups and as a statement about higher cohomology operations. The book does collect and make easily available to beginners in this subject a lot of material which was not easily obtained before. As such it is a useful addition to the literature. On the other hand it is not complete enough to be a valuable reference work and this fact may also be its biggest drawback as a text. 